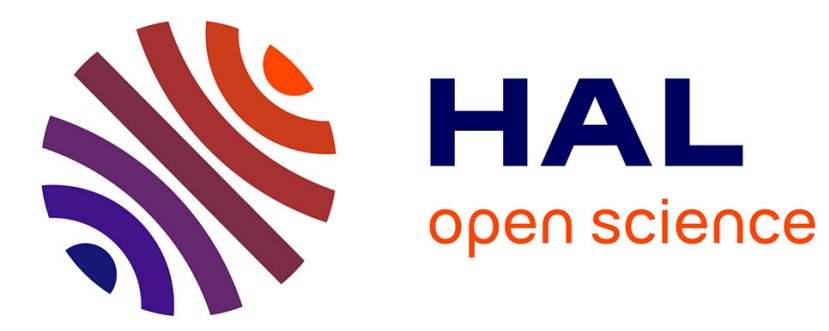

\title{
Full design of a low-cost quadrotor UAV by student team
} Jean-Baptiste Devaud, Stéphane Najko, Pierre Le Nahédic, Cédric Maussire, Etienne Zante, Julien Marzat

\section{To cite this version:}

Jean-Baptiste Devaud, Stéphane Najko, Pierre Le Nahédic, Cédric Maussire, Etienne Zante, et al.. Full design of a low-cost quadrotor UAV by student team. International Conference on System Engineering and Technology, ICSET 2012, Sep 2012, Bandung, Indonesia. hal-00732845

\section{HAL Id: hal-00732845 \\ https://hal.science/hal-00732845}

Submitted on 17 Sep 2012

HAL is a multi-disciplinary open access archive for the deposit and dissemination of scientific research documents, whether they are published or not. The documents may come from teaching and research institutions in France or abroad, or from public or private research centers.
L'archive ouverte pluridisciplinaire HAL, est destinée au dépôt et à la diffusion de documents scientifiques de niveau recherche, publiés ou non, émanant des établissements d'enseignement et de recherche français ou étrangers, des laboratoires publics ou privés. 


\title{
Full design of a low-cost quadrotor UAV by student team
}

\author{
Jean-Baptiste Devaud $^{\# 1}$, Stéphane Najko", Pierre Le Nahédic ${ }^{\#}$, Cédric Maussire", \\ Etienne Zante ${ }^{\#}$, Julien Marzat ${ }^{* 2}$ \\ ${ }^{\#}$ Students of ECE, F-75015 Paris, France \\ devauddece.fr \\ *ONERA - The French Aerospace Lab, F-91123 Palaiseau, France \\ ${ }^{2}$ julien.marzateonera.fr
}

\begin{abstract}
This paper presents the complete design of a quadrotor UAV, named VORTEX, comprising its architecture and control. The use of Unmanned Aerial Vehicles (UAV) for surveillance, observation and security gives lots of possibilities to develop new technologies. The aim of this project was to develop a low-cost modular platform under tight constraints, namely only five students and a limited budget $(450 €)$. This demonstrates the ability to build non-commercial, competitive academic platforms for control education through flying robotics.
\end{abstract}

Keywords - control education, low-cost, modular platform, quadrotor, student project, Unmanned Aerial Vehicles

\section{INTRODUCTION}

A quadrotor is an aircraft lifted and propelled by four rotors. It is considered to be a rotorcraft, as opposed to fixed-wing aircraft. The control of such a system is achieved by varying the speed of each rotor to shape the motion of the quadrotor. This kind of Unmanned Aerial Vehicle (UAV) may be useful for rescue, research and indoor or outdoor surveillance. The UAV can be closed-loop controlled so as to follow a predefined trajectory, while ensuring its stability. Automatic takeoff and landing capabilities can also be considered, as well as hovering above identified moving or stationary targets. Quadrotors are complex control systems, due to a great sensitivity in the stabilization process. Furthermore, a UAV quadrotor has a limited payload, so as to limit energy consumption.

The first quadrotor in history was developed and built by De Bothezat in 1921. Thereafter, several models were created, e.g., the Mesicopter [1]. Many studies and improvements have been conducted on quadrotor systems, which are now wellknown in the control and robotics communities. For instance, a quadrotor was modeled in [2] by incorporating the airframe and motor dynamics as well as aerodynamics and gyroscopic effects and controlled by separating the rigid body dynamics from the motor dynamics. In [3], a simple P\\&D control has been proposed for attitude stabilization. It shows how to use this kind of controller to make an acceptable stabilization of the UAV. Details concerning the aerodynamic principles of helicopters and other rotating-wing vertical lift aircraft can be found in [1]. Besides the history of helicopter flight, basic methods of analysis, and performance and design issues, this book covers airfoil flows, unsteady aerodynamics and dynamic stall, which makes it very useful for the development of rotating-wing structures. An X4-Flyer quadrotor has been conceived by a team from the Australian National University [4] to be used as a highly-reliable experimental platform. This particular project involves a lot of human and financial resources. The authors from [5] explain the different steps to conceive their micro UAV OS4, from the modeling part to the indoor control with angular orientation. The hardware and software design of the JAviator (Java Aviator), which is a high-performance quadrotor system of $1.3 \mathrm{~m}$ in diameter and a mass of $2.2 \mathrm{~kg}$ is detailed in [6]. Attempts to build fully-operational and open-source quadrotors at a moderate cost can be found in [7] or [8]. Other experimental flying platforms with vertical takeoff and landing capabilites are described in [9] and [10]. The full design of a UAV quadrotor as a platform for monitoring indoor environments is also reported in [11].

Commercial offers at competitive costs have also emerged recently. For instance, the Parrot AR Drone [12] is available for $300 €$. However, this kind of platform is usually not modular enough, since the control logic is not fully open and the UAV is not able to carry additional payload. The VORTEX project advocated in the present paper aims at showing that for a similar cost, an academic modular solution can be designed and built under restricted financial and human resources. The resulting low-cost quadrotor should be able to stabilize itself in attitude, position and have automatic takeoff and landing capabilities. This UAV will be used as an open platform for different purpose by future project teams. The visualization of the UAV motion is managed by a Human Machine Interface (HMI). This interface allows the user to switch between automatic and manual modes. The main objective of this paper is to describe the design methodology of the VORTEX system, such that other teams may follow a similar approach. Section II describes the hardware architecture, Section III and IV are dedicated to the dynamical modeling and control of the UAV and Section V to the description of the Human Machine Interface.

\section{ARCHITECTURE OF VORTEX UAV}

There is no definite version of a quadrotor: each designer, amateur or professional, has its own leading ideas. The project 
was realized by a student team of five members from ECE Paris, school of engineering. The student team responsible for this project decided to create a modular quadrotor platform for multiple uses. To reach this goal, a limited time of 500 hours (60 days of effective work) was alloted, along with a budget of $450 €$ (see Table I). The resulting UAV should be able to move inside a building, to pass through doors, to remain stable and to take off and land automatically.

TABLE I

VORTEX DETAILED BUDGET

\begin{tabular}{|l|c|c|}
\hline Designation & Quantity & Unit price \\
\hline $\begin{array}{l}\text { Motors brushless TURIGNY 1000kV } \\
200 \mathrm{~W}\end{array}$ & 4 & $10 €$ \\
\hline $\begin{array}{l}\text { Speed controllers ESC TURIGNY } \\
\text { Plush 40A }\end{array}$ & 4 & $15 €$ \\
\hline Battery LiPo 4500 mAh & 2 & $30 €$ \\
\hline Ultrasonic sensor & 1 & $20 €$ \\
\hline IMU MONGOOSE & 1 & $90 €$ \\
\hline Camera CMOS Linsprite Jpeg Color & 1 & $40 €$ \\
\hline Microcontroller MBED & 1 & $\begin{array}{c}\text { Free } \\
(\text { sponsor })\end{array}$ \\
\hline Bluetooth module & 1 & $20 €$ \\
\hline $\begin{array}{l}\text { Other materials (connectors, } \\
\text { resistors, LED, ...) }\end{array}$ & 1 & $120 €$ \\
\hline Global price & \multicolumn{4}{|c|}{} \\
\hline
\end{tabular}

First of all, the working of a quadrotor is basically the same, independently of the UAV dimensions: in order to take off, to be stable and to move, a quadrotor needs two different pairs of propellers rotating clockwise and counter-clockwise, as shown in Fig. 1.

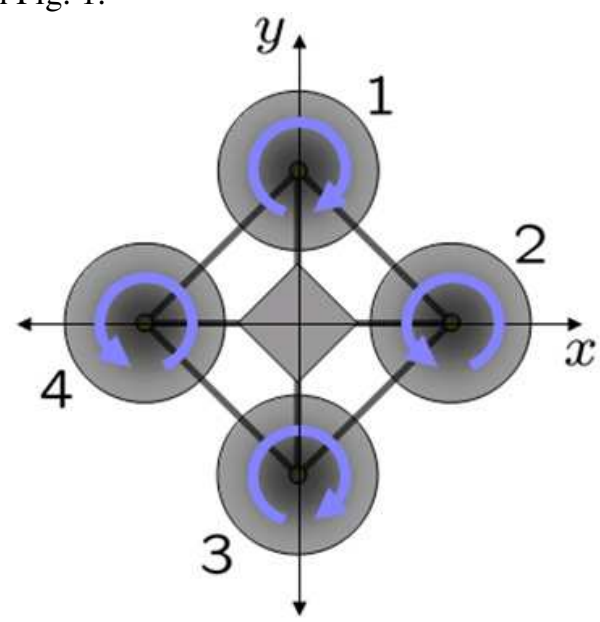

Fig. 1 Turning directions of rotors

In this section of the paper, the different parts of the architecture of the quadrotor are detailed, namely the mechanical construction of the quadrotor VORTEX and the hardware choice of electronic components and sensors.

\section{A. Mechanical Structure}

The design of the quadrotor VORTEX based itself on a classical cross structure (Fig. 2). At the center, a circular platform was fixed in order to place the control card of the UAV. The material chosen for the structure is aluminum, since it is light, malleable while resilient and easy to work. This seemed to be a more appropriate choice than carbon fiber, which is tougher and harder to machine. The cross structure can also be repared more easily if aluminum bars are used, since working carbon fiber is really expensive and thus does not fit with the spirit of this low-cost project.

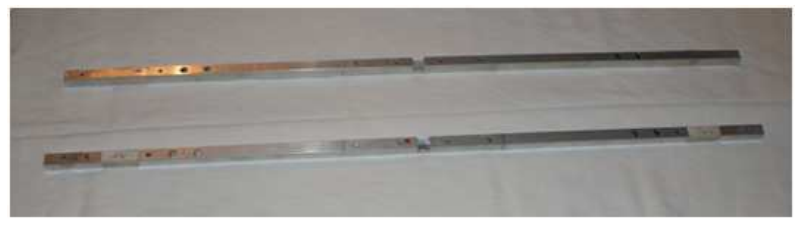

Fig. 2 Axes for cross structure

The central platform is also built in aluminum and realized by stamping. This is where the electronic card and the controllers for the four engines were fixed, as shown on Fig. 3.
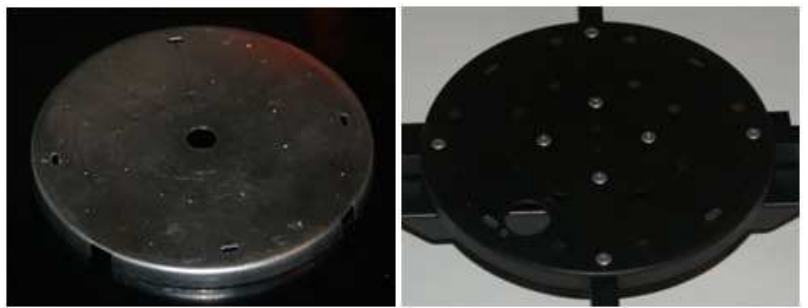

Fig. 3 Platform (prototype and final version)

The protection, composed of a double circle with intermediate links, is also made of aluminum. This structure is fixed on the four arms of the quadrotor, as can be seen on Fig. 4.

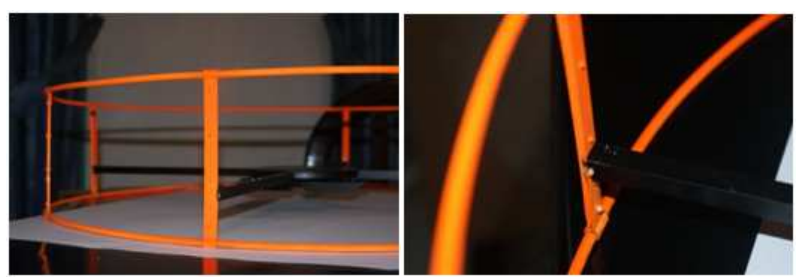

Fig. 4 Protection

Four stands were also designed to check ground contact, in order to help landing (Fig. 5).

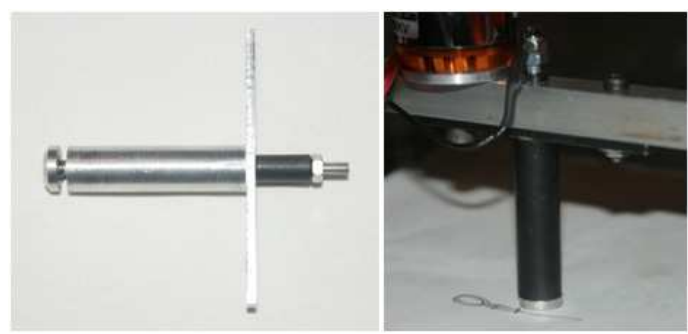

Fig. 5 Stands (prototype and final version)

Quadrotor VORTEX has a full aluminum structure resulting in a total weight of $324 \mathrm{~g}$. It includes the two axis of the cross structure, the central platform, the circular protection and the battery slot beneath the platform, as shown on Fig. 6 . 


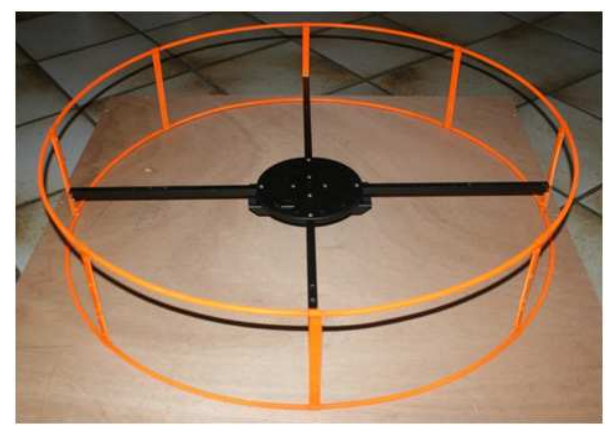

Fig. 6 UAV structure

The protection of the electronic part of the quadrotor is provided by a plastic dome (see Fig. 7). It isolates the electronic part from the outside and prevents risks of damages.

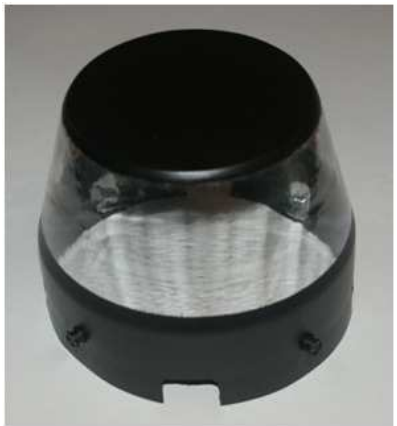

Fig. 7 Protection dome

The overall dimensions are finally a diameter of $61 \mathrm{~cm}$, a height of $14 \mathrm{~cm}$ for an overall mass of $1.4 \mathrm{~kg}$. The fullyassembled quadrotor is displayed in Fig. 8.

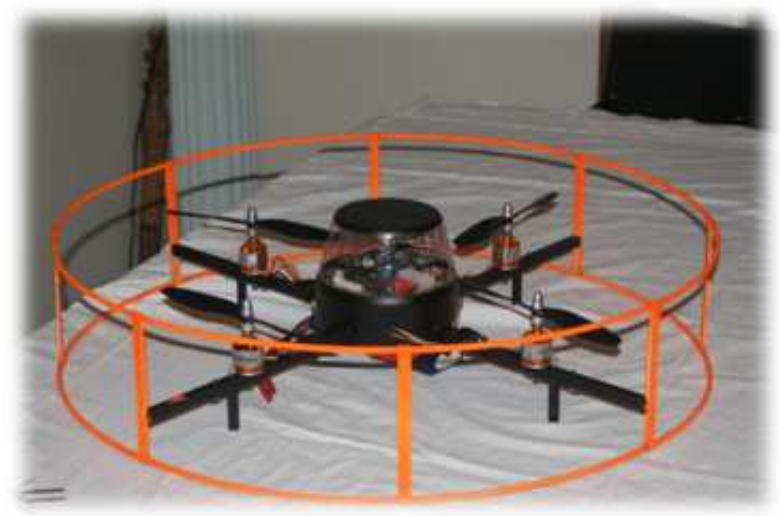

Fig. 8 Assembled quadrotor

\section{B. Hardware Structure}

The electronic routing and the central electronic card of the system were designed by the project team. It centralizes all the data from the different sensors and components of the system, which are listed below.

- A Mbed card with an ARM Cortex-M3 Microcontroller. This component centralizes all the data from the different sensors in our system.

- An Inertial Measurement Unit (IMU) Mongoose 9DOF. This component measures the non-gravitational accelerations on the three axis and the angular velocities in yaw, pitch and roll. A three-axis magnetometer provides additional information for position and attitude estimation.

- An AtXmega controller. This component is used in order to control the communication between the different elements of the electronic card.

- A Bluetooth module RN-41 for the communication between the quadrotor and the laptop unit.

- An ultrasonic range finder MaxSonar. This component is used to measure the altitude of the quadrotor.

The system is powered by a LiPo Battery of $4500 \mathrm{mAh}$, which makes possible a flight of at least 15 minutes. Fig. 9 shows the power supply chain of the UAV, while Fig. 10 presents the electronics.

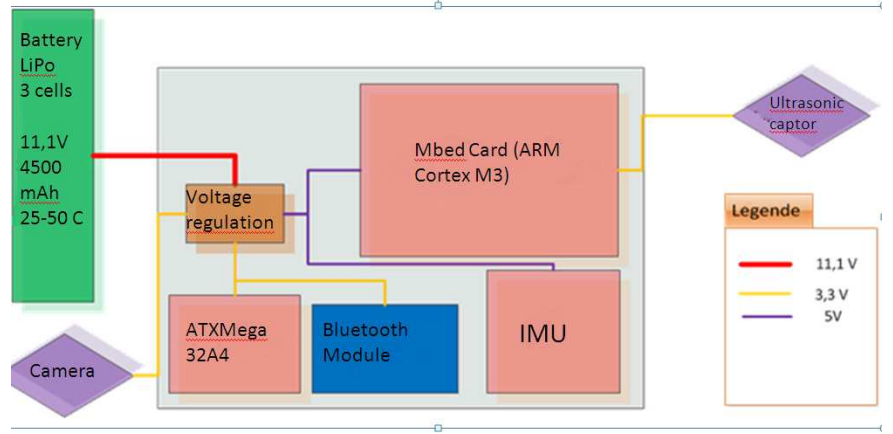

Fig. 9 Power supply chain

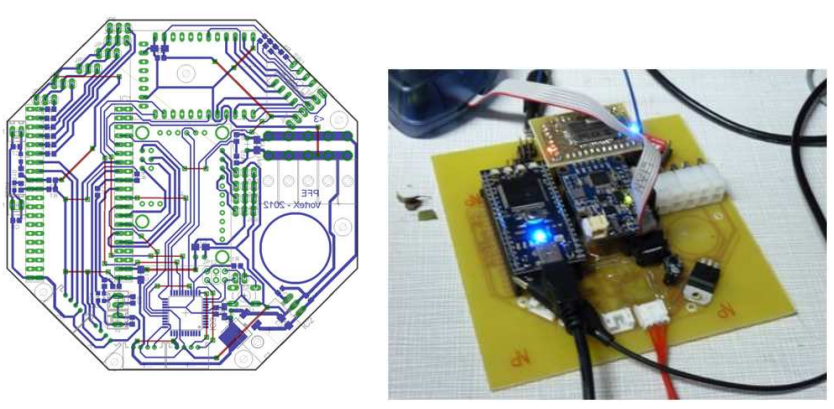

Fig. 10 Electronics

\section{DYNAMICAL MODELING}

The quadrotor should be closed-loop controlled to ensure its stabilization and to be able to follow autonomously a given trajectory. The motion of the quadrotor is governed by the adjustment of the relative values of the four rotor speeds. These control inputs have direct effects on the three angles and angular velocities in yaw $(\psi, r)$, pitch $(\theta, q)$ and roll $(\phi, p)$. For takeoff, the four rotor speeds can be increased with the same value (Fig. 11.a). To move the UAV to the right or to the left, the values of the left and right rotors should be modified. This operation has an impact on the value of the roll angle (Fig. 11.b). The forward motion is obtained by increasing the speed of the front rotor while decreasing the rear rotor speed. This operation changes the pitch angle (Fig. 11.c). The third motion on the yaw angle is obtained by 
increasing the counter-clockwise rotors speed while decreasing the clockwise rotor speed (Fig. 11.d).

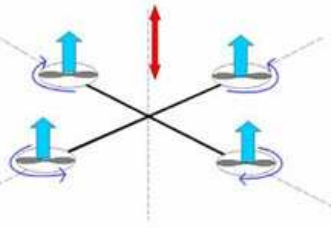

(a) Main thrust

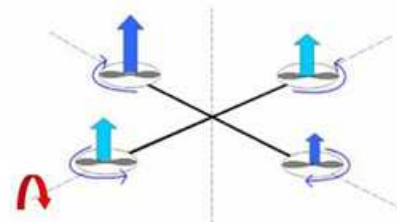

(c) Pitch angle

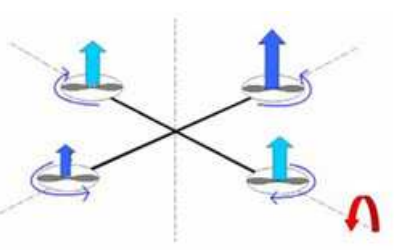

(b) Roll angle

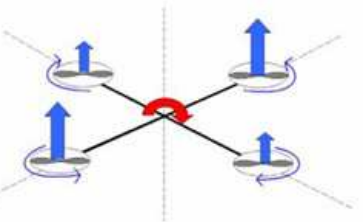

(d) Yaw angle
Fig. 11 Quadrotor motion

For dynamical modeling, the convention axis from Fig. 12 is adopted. In this scheme, the frame $I=\left\{e_{X}, e_{Y}, e_{Z}\right\}$ is an inertial frame linked to the ground. The body frame $A=\left\{e_{1}, e_{2}, e_{3}\right\}$ is attached to the quadrotor axes. The three angles around the axes of this frame define the three rotations in roll $(\phi)$, pitch $(\theta)$ and yaw $(\psi)$.

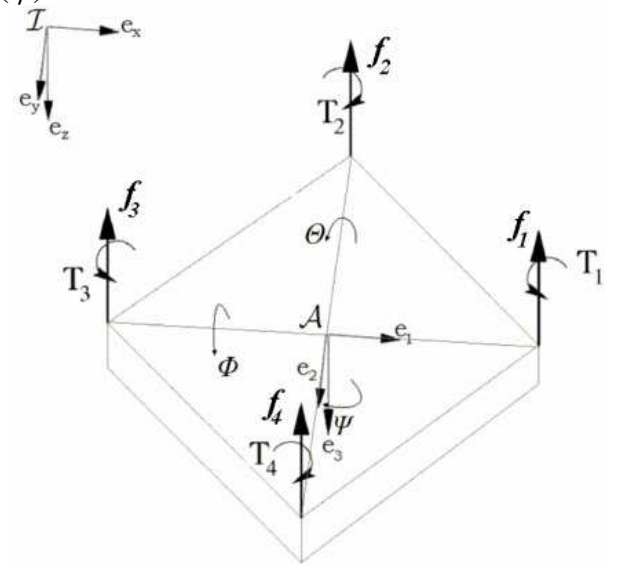

Fig. 12 Body frame definition

The dynamics of the quadrotor system is well-established, and can be summarized by the six following equations, composing the force and torque equations [13].

$$
\left\{\begin{array}{c}
\ddot{x}=\left(\frac{\cos \psi \sin \theta \cos \phi+\sin \psi \sin \phi}{m}\right) \sum_{i=1}^{4} T_{i} \\
\ddot{y}=\left(\frac{\sin \psi \sin \theta \cos \phi+\cos \psi \sin \phi}{m}\right) \sum_{i=1}^{4} T_{i} \\
\ddot{z}=-g+\left(\frac{\cos \theta \cos \phi}{m}\right) \sum_{i=1}^{4} T_{i}
\end{array}\right.
$$

$$
\left\{\begin{array}{c}
\ddot{\phi}=\dot{\theta} \dot{\psi}\left(\frac{I_{Y}-I_{Z}}{I_{X}}\right)+b l\left(\frac{\omega_{4}^{2}-\omega_{2}^{2}}{I_{X}}\right)+\dot{\theta} I_{\text {rotor }}\left(\frac{\omega_{1}-\omega_{2}+\omega_{3}-\omega_{4}}{I_{X}}\right) \\
\ddot{\theta}=\dot{\psi} \dot{\phi}\left(\frac{I_{Z}-I_{X}}{I_{Y}}\right)+b l\left(\frac{\omega_{3}^{2}-\omega_{1}^{2}}{I_{Y}}\right)+\dot{\phi} I_{\text {rotor }}\left(\frac{\omega_{1}-\omega_{2}+\omega_{3}-\omega_{4}}{I_{Y}}\right) \\
\ddot{\psi}=\dot{\phi} \dot{\theta}\left(\frac{I_{X}-I_{Y}}{I_{Z}}\right)+d\left(\frac{-\omega_{1}^{2}+\omega_{2}^{2}-\omega_{3}^{2}+\omega_{4}^{2}}{I_{Z}}\right)
\end{array}\right.
$$

In these equations, $T_{i}$ is the thrust of the rotor force $f_{i}$ and $\omega_{i}$ the rotation speed of the corresponding rotor. The 3D position of the UAV in the inertial frame is provided by $[x, y, z]$. The inertia matrix of the vehicle is diagonal, due to symmetry. Its coefficients $\left[I_{X}, I_{Y}, I_{Z}\right]$ have been determined using the $3 \mathrm{D}$ structural modeling presented in Fig. 13. Table II summarizes the value of all the quadrotor parameters.

Since the parameters $b$ (drag coefficient), $d$ (range coefficient) and $I_{\text {rotor }}$ (Rotor inertia) were unknown or very uncertain, they needed to be estimated from flight data. Preliminary proportional controllers have been used to obtain basic stabilization and thus collect a few seconds of flight. The resulting IMU data has then been exploited to identify these three parameters $\left[b, d, I_{\text {rotor }}\right]$. This can be achieved by rewritting the system of equations (2) as

$$
\left[\begin{array}{c}
\ddot{\phi}-\dot{\theta} \dot{\psi}\left(\frac{I_{Y}-I_{Z}}{I_{X}}\right) \\
\ddot{\theta}-\dot{\psi} \dot{\phi}\left(\frac{I_{Z}-I_{X}}{I_{Y}}\right) \\
\ddot{\psi}-\dot{\phi} \dot{\theta}\left(\frac{I_{Z}-I_{X}}{I_{Y}}\right)
\end{array}\right]=\left[\begin{array}{ccc}
h_{11} & 0 & h_{13} \\
h_{21} & 0 & h_{23} \\
0 & h_{32} & 0
\end{array}\right]\left[\begin{array}{c}
b \\
d \\
I_{\text {rotor }}
\end{array}\right]
$$

where

$$
\left\{\begin{array}{c}
h_{11}=l\left(\frac{\omega_{4}^{2}-\omega_{2}^{2}}{I_{X}}\right) \\
h_{13}=\dot{\theta}\left(\frac{\omega_{1}-\omega_{2}+\omega_{3}-\omega_{4}}{I_{X}}\right) \\
h_{21}=l\left(\frac{\omega_{3}^{2}-\omega_{1}^{2}}{I_{Y}}\right) \\
h_{23}=\dot{\phi}\left(\frac{\omega_{1}-\omega_{2}+\omega_{3}-\omega_{4}}{I_{Y}}\right) \\
h_{32}=\frac{-\omega_{1}^{2}+\omega_{2}^{2}-\omega_{3}^{2}+\omega_{4}^{2}}{I_{Z}}
\end{array}\right.
$$




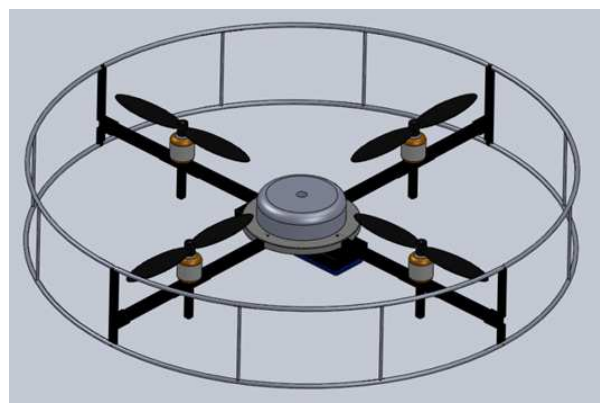

Fig. 13 Quadrotor 3D modeling

Since this system is linear, the unknown parameters can be estimated via classical least square from available filtered flight data [14]. Fig. 14 shows the correspondence between real flight data and the simulated model with the estimated parameters.

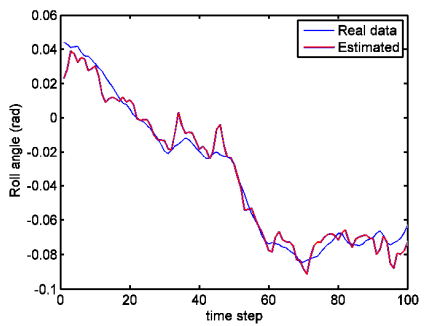

1) Roll angle

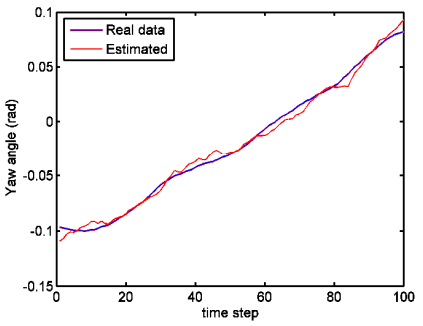

2) Yaw angle
Fig. 14 Identified model compared to flight data

TABLE III

VORTEX QUADROTOR PARAMETERS

\begin{tabular}{|c|c|c|}
\hline Parameter & Notation & Value \\
\hline Half-length & $l$ & $0.305 \mathrm{~m}$ \\
\hline Drag coefficient & $b$ & $1.810^{-6}$ \\
\hline Range coefficient & $d$ & $7.110^{-7}$ \\
\hline Moment of inertia of a rotor & $I_{\text {rotor }}$ & $3.510^{-4} \mathrm{~kg} / \mathrm{m}^{2}$ \\
\hline Mass & $m$ & $1.4 \mathrm{~kg}$ \\
\hline Moment of inertia on x axis & $I_{X}$ & $1.03510^{-2} \mathrm{~kg} / \mathrm{m}^{2}$ \\
\hline Moment of inertia on y axis & $I_{Y}$ & $1.6610^{-2} \mathrm{~kg} / \mathrm{m}^{2}$ \\
\hline Moment of inertia on z axis & $I_{Z}$ & $7.4310^{-2} \mathrm{~kg} / \mathrm{m}^{2}$ \\
\hline Roll proportional gain & $k_{p, \phi}$ & 62 \\
\hline Roll derivative gain & $k_{d, \phi}$ & 700 \\
\hline Pitch proportional gain & $k_{p, \theta}$ & 62 \\
\hline Pitch derivative gain & $k_{d, \theta}$ & 700 \\
\hline Yaw proportional gain & $k_{p, \psi}$ & 110 \\
\hline Yaw derivative gain & $k_{d, \psi}$ & 700 \\
\hline Altitude proportional gain & $k_{p, z}$ & 25 \\
\hline Altitude derivative gain & $k_{d, z}$ & 75 \\
\hline
\end{tabular}

where $T_{\text {floor }}$ is a thrust value that has been found experimentally to compensate for the weight of the UAV. An additional PID controller in altitude was also developed in order to allow automatic takeoff and landing. For these procedures, the altitude measurement is provided by the ultrasonic range finder. The takeoff algorithm is quite simple: the speeds of the four rotors are increased progressively in order to reach $T_{\text {floor }}$ then the altitude reference is fixed to one meter (for instance), for the UAV to reach it. The landing procedure first proceeds by fixing the altitude reference to 20 centimeter. The rotor speeds are then gradually decreased until the UAV touches the ground. The rotors are stopped when at least one stand (Fig. 5) is activated. A sequence of stable flight with this control strategy is displayed in Fig. 16. 

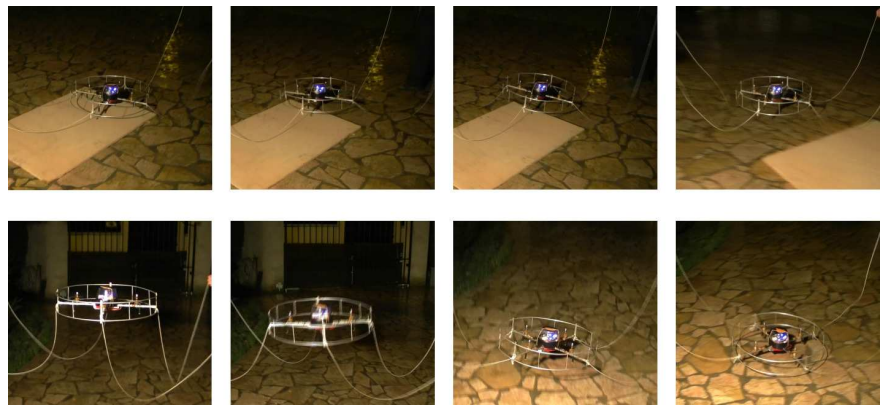

Fig. 16 Flight sequence

\section{HUMAN MACHINE INTERFACE}

The quadrotor VORTEX is connected to a laptop to keep a human supervision on the UAV (Fig. 17). This HMI is split up into four parts.

1) Flight data: this part displays the angles of roll, pitch and yaw with flight time, altitude, pressure, temperature, the four contacts and the battery level. A 3D visualization of the UAV attitude is also available.

2) Communication: this is where the raw data are displayed. The user connects the laptop to the UAV with the Bluetooth connexion in order to activate it and to receive data from the quadrotor.

3) Video: this part displays the video filmed by the embedded camera.

4) UAV control: this is the part used to send orders to the UAV. Takeoff or landing procedures can be activated, along with motion orders. It is possible to switch between automatic and manual modes.

\section{CONCLUSIONS}

In this project, the members of a student team developed a low-cost quadrotor UAV, VORTEX, with basic hardware and electronic components. The UAV should be able to move into buildings or in a urban environment. The main electronic card that centralizes all the data was self-designed. All the elements (IMU, ultrasonic captor, Mbed...) were plugged on this card. Classical P\&D control was called upon to stabilize the UAV in roll, pitch and yaw and to reach a reference altitude. Two simple procedures for automatic takeoff and landing were also implemented. A Human Machine Interface (HMI) was developed in order to collect data and to switch between automatic and manual modes.

The quadrotor is finally operational: the correction in roll, pitch and yaw angles is functional, the HMI can communicate with the quadrotor and orders can be sent, takeoff and landing procedures are implemented. The VORTEX project has shown the feasibility of building low-cost open UAV platforms for control education, with limited resources. Thanks to this first step, additional sensors and more elaborate control strategies may then be experimented by future student teams.

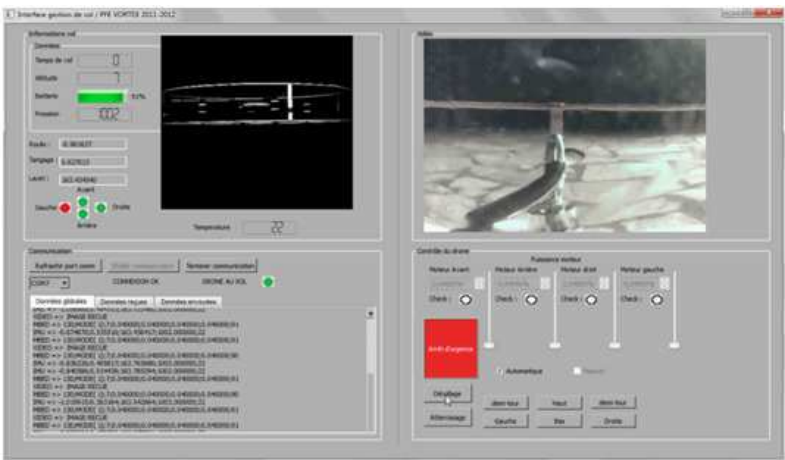

Fig. 17 User interface

\section{ACKNOWLEDGMENT}

The authors would like to thank ECE Paris for their support to this project.

\section{REFERENCES}

[1] J. Leishman, Principles of Helicopter Aerodynamics, Cambridge University Press, New York, 2006.

[2] T. Hamel, R. Mahony, R. Lozano and J. Ostrowski, "Dynamic modeling and configuration stabilization for an X-4-flyer" in Proceedings of the $15^{\text {th }}$ IFAC World Congress, Barcelona, Spain, 2002.

[3] B. Erginer and E. Altug, "Modelling and PD control of a quadrotor VTOL vehicle" in Proceedings of the IEEE Intelligent Vehicles Symposium, Istanbul, Turkey, pp. 894-899, 2007.

[4] P. Pounds, R. Mahony and P. Corke, "Modelling and control of a quadrotor robot" in Proceedings of the Australasian Conference on Robotics and Automation, Auckland, New Zealand, 2006.

[5] S. Bouabdallah, P. Murrieri, and R. Siegwart, "Design and control of an indoor micro quadrotor" in Proceedings of the IEEE International Conference on Robotics and Automation, ICRA'04, New Orleans, USA, vol. 5, pp. 4393-4398, 2004.

[6] S. Craciunas, C. Kirsch, H. R"ock, and R. Trummer, "The JAviator: A high-payload quadrotor UAV with high-level programming capabilities" in Proceedings of the 2008 AIAA Guidance, Navigation and Control Conference, Honolulu, Hawaii, 2008.

[7] S. Hanford, L. Long, and J. Horn, "A small semi-autonomous rotarywing unmanned air vehicle (UAV)" AIAA InfoTech \& Aerospace Conference, Washington D.C., USA, 2005.

[8] L. K. Burkamshaw, Towards a Low-Cost Quadrotor Research Platform, Master Thesis of Naval PostGraduate School, Monterey, California, 2010.

[9] K. Weng and M. Abidin, "Design and control of a quad-rotor flying robot for aerial surveillance" in Proceedings of the 4th IEEE Student Conference on Research and Development, Shah Alam, Malaysia, pp. 173-177, 2006.

[10] B. Herisse, F. Russotto, T. Hamel, and R. Mahony, "Hovering flight and vertical landing control of a VTOL unmanned aerial vehicle using optical flow" in Proceedings of the IEEE International Conference on Intelligent Robots and Systems, Nice, France, pp. 801-806, 2008.

[11] B. Min, C. Cho, K. Choi, and D. Kim, "Development of a micro quadrotor uav for monitoring an indoor environment" Advances in Robotics, vol. 5744, pp. 262-271, 2009

[12] P. Bristeau, F. Callou, D. Vissi`ere, and N. Petit, "The navigation and control technology inside the ar. drone micro UAV" in Proceedings of the 18th IFAC World Congress, Milano, Italy, pp. 1477-1484, 2011.

[13] P. Castillo, A. Dzul, and R. Lozano, "Real-time stabilization and tracking of a four-rotor mini rotorcraft," IEEE Transactions on Control Systems Technology, vol. 12, no. 4, pp. 510-516, 2004.

[14] E. Walter and L. Pronzato, Identification of Parametric Models from Experimental Data. Springer Verlag, London, 1997.

[15] M. Euston, P. Coote, R. Mahony, J. Kim, and T. Hamel, "A complementary filter for attitude estimation of a fixed-wing UAV" in Proceedings of the International Conference on Intelligent Robots and Systems, IROS'08, Nice, France, pp. 340-345, 2008. 